\title{
NOTA SOBRE SURTO DE MALÁRIA EM USUÁRIOS DE DROGAS INJETÁVEIS
}

\author{
Luiz Carlos Barradas Barata* \\ Maria Tereza Macoris Andriguetti* \\ Marcia Caraça Cortás** \\ Cleusa Meneguetti***
}

BARATA, L. C. B. et al. Nota sobre surto de malária em usuários de drogas injetáveis. Rev. Saúde públ., S.Paulo, 24: 321-2, 1990.

RESUMO: Relata-se surto de malária por Plasmodium vioax entre usuários de drogas injetáveis, detectado na cidade de Bauru, Estado de São Paulo, Brasil. Até julho de 198912 casos haviam sido confirmados pelo exame de gota espessa, e 20 outros contactantes suspeitos estavam sendo investigados. Todos os casos relataram uso freqüente de cocaína injetável, compartilhando seringas e agulhas, e negaram viagens por áreas endêmicas de malária.

DESCRITORES: Malária, epidemiologia. Abuso de substâncias. Cocaína.

A primeira referência à malária induzida, entre usuários de drogas injetáveis, data de 1929 e, desde então, vários casos têm sido relatados em outros países ${ }^{1,2,3,5}$.

No Brasil, apesar do número crescente de casos de malária registrados nas últimas décadas ${ }^{4}$, a ocorrência da doença por essa categoria de transmissăo năo tinha sido assinalada até recentemente.

Em agosto de 1988, um surto de malária por $P$. vivax entre usuários de cacaína endovenosa foi detectado na cidade de Presidente Prudente, SP, tendo sido registrados 9 casos e 3 reinfecçరes****. Nesse mesmo ano, 2 outros casos de malária por $P$. vivax em usuários de drogas injetáveis foram notificados no Município de Săo Paulo, SP.

Em julho de 1990 um surto de malária induzida em usuários de cocaína injetável foi assinalado na cidade de Bauru, Estado de São Paulo, Brasil, tendo sido notificados, até o momento, 12 casos e 20 contactantes, sendo que 14 ainda não foram localizados. Todos os casos detectados tiveram infecção por $P$. vivax, confirmada pelo meio de exame hemoscópico pela técnica de gota espessa, realizada pelo Laboratório Regional da Superintendência de Controle de Endemias (SUCEN). Os pacientes são todos do sexo masculino, com idades entre 18 e 27 anos, residentes na cidade de Bauru. As investigaçōes epidemiológicas desses casos revelaram que todos faziam uso de cocaína injetável, compartilhando seringas e agulhas e que nenhum deles havia tido malária anteriormente ou havia estado em áreas de transmissão da doença nos últimos 18 meses. Dos 12 casos detectados até o momento, 8 residem em uma mesma localidade da periferia de Bauru, os demais resi-dem em outros bairros da cidade.

O receio de que a confirmação diagnóstica e de que as informaçōes prestadas possam ser utilizadas em processos judiciais tem dificultado a identificação e tratamento dos envolvidos. Em razão disso a SUCEN optou por tratar com cloroquina todos os indivíduos que tenham feito uso em comum de seringas ou agulhas com os casos confirmados ou com seus contactantes, independentemente da existência ou não de sintomas sugestivos de malária ou do resultado dos exames hemoscópicos.

A existência de casos confirmados entre indivíduos de distintos extratos sociais e residentes em diferentes bairros da cidade sugere que a transmissão da malária possa estar ocorrendo em diversos círculos de usuários de drogas injetáveis no $\mathrm{Mu}$ nicípio de Bauru.

A ocorrência de outras doenças infecciosas veiculadas pelo uso comum de seringas ou agulhas será avaliada mediante realização de provas sorológicas específicas realizadas em amostras de sangue coletadas dos envolvidos.

- Superintendência de Controle de Endemias - SUCEN - Rua Paula Souza, 166 - 01027 - São Paulo, SP - Brasil.

* Centro de Vigilância Epidemiológica - Secretaria de Estado da Saúde - Av. São Luiz, 99 - 01046 - São Paulo, SP. - Brasil.

** Escritório Regional de Saúde (ERSA) de Bauru - Rua Antonio Zuiaru, Q.5, s/n² - 17100 - Bauru, SP - Brasil.

*** LO, S. S. et al. Malária induzida por droga injetável associada à soropositividade para HIV em Presidente Prudente, SP. Dados inéditos. 
BARATA, L. C. B. et. al [Note on the malaria outbreak in users of injectable drugs]. Rev. Saúde públ., S.Paulo, 24: 321-2, 1990

ABSTRACT: An outbreak of Plasmodium vioax malaria among drug addicts in Bauru, S. Paulo State, Brazil, is reported. Until July 1989, 12 cases had been confirmed by thick smear examinations and 20 contacts were being investigated. All of them reported frequent use of intravenous cocaine and sharing of needles and syringes but deny having travelled through endemic malarial areas.

KEYWORDS: Malaria, epidemiology. Substance abuse. Cocaine.

\section{REFERENCIAS BIBLIOGRAFICAS}

1. BIGGAM, A. G. Malignant malaria associated with the administration of heroin intravenously. Trans, roy. Soc. trop. Med. Hyg, 23: 147-53, 1929.

2. BROWN, J. D. \& KHOA, N. Q. Fatal falciparum malaria among narcotic injects. Amer. J. trop. Med. Hyg., 24: $729-33,1975$.

3. FRIEDMANN, C. T. H.; DOVER, S. A ; ROBERTO, R. R. A malaria epidemic among heroin users. Amer.
J. trop. Med. Hyg., 22: 302-7, 1973.

4. MARQUES, A. C. Human migration and the spread of malaria in Brazil. Parasit. Today, 3: 166-70, 1987.

5. ROSEMBLATT, J. E. \& MARSH, V. H. Induced malaria in narcotic addicts. Lancet, 2: 189-91, 1971.

Recebido para publicação em 7/8/1990 Aprovado para publicação em 8/9/1990 